\title{
B2 SINE retrotransposon causes polymorphic expression of mouse 5-aminolevulinic acid synthase 1 gene
}

Tatyana Chernova, Fiona M. Higginson, Reginald Davies, Andrew G. Smith*

MRC Toxicology Unit, University of Leicester, Leicester, UK

*Corresponding author. FAX: +44 1162525616

E-mail address: ags5@le.ac.uk (A.G. Smith)

Full address:

MRC Toxicology Unit,

Hodgkin Building,

University of Leicester

P.O. Box 138

Lancaster Road

Leicester LE1 9HN

UK 


\begin{abstract}
5-Aminolevulinic acid synthase 1 (ALAS1) is the key enzyme in the homeostasis of nonerythroid heme and of fundamental importance in respiration, the metabolism of drugs, chemicals and steroids and cell signalling. The regulation of ALAS1 in response to stimuli occurs at transcriptional, translational and post-translational levels which could depend on inter-individual variation in basal expression. A genetic difference in hepatic ALAS1 mRNA levels between C57BL/6J and DBA/2 mice was detected by microarray and was $>5$-fold in whole liver or hepatocytes when estimated by qRT-PCR. Analysis of the ALAS1 promoter showed a $210 \mathrm{nt}$ insert in the DBA/2 containing a B2 SINE retrotransposon causing a marked repression of expression by intracellular reporter systems. Deletions across the B2 SINE demonstrated that the full sequence was required for transcriptional inhibition. The findings show that a B2 SINE can contribute to the regulation of ALAS1 and SINEs in 5'-UTR regions contribute to inter-individual differences in gene expression.
\end{abstract}

Keywords: 5-Aminolevulinic acid synthase 1; promoter; B2 SINE; polymorphism. 
Heme has a fundamental role in biology serving as the prosthetic group for a large range of catalytic proteins including respiratory cytochromes, chemical, drug and steroid metabolism enzymes, catalase, peroxidases and nitric oxide synthases [1]. The ability of heme to bind gases reversibly accounts for its functions in other hemoproteins in transporting oxygen, and as a sensor of $\mathrm{O}_{2}, \mathrm{NO}$ and $\mathrm{CO}$ [2]. Heme also has many roles in the control of gene expression and cellular metabolism as a signalling molecule modulating the activity of ion channels, transcription factors and kinases [3,4], microRNA processing [5] and the N-rule pathway of protein degradation [6].

The supply of heme is dependent on the availability of 5-aminolevulinic acid (ALA) the first intermediate in the biosynthetic pathway. Nonerythroid ALA synthase (ALAS1) is a key enzyme in liver and other tissues and its synthesis is controlled, in part, via negative feedback of a proposed regulatory heme pool by a variety of mechanisms including inhibition of translocation of the pre-protein into mitochondria, stability of ALAS1 mRNA and repression of $A L A S 1$ gene transcription [1]. Variation in the core $A L A S 1$ promoter may be responsible for tissue specific expression $[7,8]$. ALAS1 expression is regulated not only by heme but by a number of regulatory 5'-UTRs responding to cellular messengers including those associated with drug response, nutrition and circadian rhythm [9-13].

Inter-individual variation in gene expression of heme metabolism enzymes has potential in modulating heme supply. This could be particularly pertinent to disorders in which heme supply is genetically or chemically disturbed (porphyrias). A difference between the inbred mouse strains in the gene dosage of 5-aminolevulinate dehydratase may contribute to responses to lead poisoning [14]. Although low heme availability can lead to feed-back derepression of $A L A S 1$ transcription it is probably triggered depending on the level of basal expression. Polymorphisms in ALAS1 response to stimuli, such as seen with chemicals and drugs that cause porphyria, might be the reflection of variation in basal expression $[15,16]$. 
Recently, the exciting potential of non-coding RNAs to regulate cellular functions, including transcription, has become an important paradigm in understanding gene regulation [17]. Stress induces RNA formation from the B2 short interspersed nuclear element (SINE) retrotransposon which has been shown to bind to RNA polymerase II (Pol II) and repress transcription of genes $[18,19]$. Retrotransposons may also act in cis regulatory exaptation mechanisms such as mRNA polyadenylation, alternative splicing and in promoter and enhancer activities [20].

We sought to determine whether variability in the 5'-UTR might account for genetic dependent basal expression of the ALAS1 gene in mice. We observed a marked difference in constitutive liver levels of ALAS1 mRNA between C57BL/6J and DBA/2 inbred mouse lines. A B2 SINE retrotransposon was identified in the promoter region of the DBA/2 ALAS1 gene inhibiting transcription. The introduction of a B2 SINE repeat in ALAS1 promoter contributes to overall polymorphic control of transcription of the gene between the two strains.

\section{Materials and methods}

Mice. Young male C57BL/6JOla and DBA/2Ola mice were purchased from Harlan Ltd, Bicester, UK and maintained on RM1 diet. Mice were killed between 10 and 11 am in all studies to avoid any apparent variation between strains due to circadian rhythm.

Hepatocyte isolation. Hepatocytes were isolated from C57BL/6J and DBA/2 mice by collagenase perfusion as described previously [21] and cultured in MEM supplemented with non-essential amino acids (Invitrogen, Paisley, UK), 2mM glutamine and 10mM penicillin/ streptomycin.

Sequencing. Fragments of approximately $500 \mathrm{nt}$ were amplified using Extensor Hi-Fidelity PCR Master Mix (Thermo Scientific, Epsom, UK), and Peltier Thermal Cycler (Bio-Rad Life 
Sciences, Hercules, USA), purified using Qiaquick PCR purification columns (Qiagen, Crawley, UK) and sequenced using an Applied Biosystems 3730 sequencer.

Black 6 and DBA promoter cloning. Fragments containing up to $\sim 2000 \mathrm{nt}$ from the 5'UTR of C57BL/6J and DBA/2 strains were amplified to produce the following constructs: 2MPR (-1229 to +82 nt) using primers MPRF2 and MPRR2; MPRM (-1888 to +82 nt) using primers MPRmiF and MPRR2; MPREx2 (-1229 to + $220 \mathrm{nt})$ using primers MPRF2 and MPREx2R; DBA222 and BL6 ( -993 to $+81 \mathrm{nt})$ using primers $222 \mathrm{~F}$ and $222 \mathrm{R}$. These were cloned into pGL3 Basic vector (Promega). The DBA/222 construct was used for further systematic deletions using primers shown in Table 1. The identities of the products were checked by sequencing.

Transfections and luciferase assays. $0.5 \mu \mathrm{g}$ pGL3 clones were transfected into 293 HEK cells using GeneJammer transfection reagent (Stratagene, La Jolla, CA). In addition $0.1 \mu \mathrm{g}$ pRL-cmv plasmid (Promega) was co-transfected as a control for transfection efficiency. After $24 \mathrm{hr}$ the luciferase activity produced by both plasmids was assayed using Dual Luciferase Assay Kit (Promega, Southampton, UK). The activity of the test plasmid normalised against the control was used as an indication of the promoter activity. Transfections were carried out a minimum of 3 times. Phenobarbital was obtained from Sigma-Aldrich, Poole, UK. 1,4-Bis[2-(3,5-dichloropyridyloxy)]benzene (TCPOBOP) was a gift from Dr D. Dinsdale.

TCPOBOP Stock solution was prepared in DMSO, then diluted in medium to final concentration $10 \mu \mathrm{M}$, used for 24 hour treatment. PB Stock solution was prepared in sterile saline used at final concentration $500 \mu \mathrm{M}$ for 24 hour treatment. 
Gene expression analysis by microarray. Preliminary comparison of expression of heme synthesis genes and the significance was carried out by gene array analysis as reported by Davies et al. using arrays of candidate ESTs [22].

Quantitative real-time PCR ( $q R T-P C R)$. qRT-PCR of ALAS1 mRNA was performed as described previously with $\beta$-actin as the reference gene [4].

Primers sequences were as follows:

$\beta$-actin forward primer 5'-GATTACTGCTCTGGCTCCTAGCA-3', reverse primer 5'GTGGACAGTGAGGCCAGGAT-3';

ALAS1 forward primer 5'-TCTTCCGCAAGGCCAGTCT-3', reverse primer 5'TGGGCTTGAGCAGCCTCTT-3'.

Statistics. Statistical significance was estimated using the two-tailed Student's $t$ test.

\section{Results and discussion}

Polymorphism of ALAS1 mRNA levels.

Initial comparison of the hepatic expression of genes in C57BL/6J and DBA/2 mice for enzymes of nonerythroid heme synthesis using gene microarrays showed the established strain-dependant expression of the ALA dehydratase gene due to gene dosage [14]. In addition, levels of ALAS1 mRNA were significantly less in DBA/2 than in C57BL/6J (Table 2). The strain difference in the level of ALAS1 mRNA between C57BL/6J and DBA/2 mice was confirmed by qRT-PCR. Levels were approximately 5-fold greater in C57BL/6 J compared with DBA/2 for whole liver (Fig. 1A) and freshly isolated hepatocytes (Fig. 1B). Search for a polymorphism in the 5'-UTR

To find out whether the polymorphic expression could be explained by differences between the strains in the promoter region of the ALAS1 gene, the 5'-UTR sequences ranging from $\sim 2000 \mathrm{nt}$ to the translation initiation codon in exon 2 were amplified from DBA/2 and 
C57BL/6J genomic DNA and cloned in a luciferase reporter vector which was used to transfect human embryonic kidney 293 cells (Fig. 2A). The greatest promoter activity detected was in the cells transfected with the 2 MPR construct (-1229 to +82 nt) (Fig. 2 B) which contains the ALAS1 core promoter. Neither in HEK 293 cells or HEP 2 cells was the promoter activity of the 2MPR construct significantly sensitive to phenobarbital or TCPOBOP (Fig. 2C) known to up-regulate ALAS1 expression by mechanisms elsewhere in the 5'-UTR $[9,10]$. The comparison of the two PCR products for 2MPR from the two strains showed that amplified from DBA/2 was larger by about $200 \mathrm{nt}$ (Fig. 2D). Sequencing of the 2MPR constructs from the two strains revealed a $210 \mathrm{nt}$ insert at $-702 \mathrm{nt}$ in the core promoter of $\mathrm{DBA} / 2$.

Two constructs containing the DNA fragment with and without the 210 nt extra-sequence were made to analyse their promoter activity. Comparison of refined constructs of the C57BL/6 region -989 to $+81 \mathrm{nt}$ with the $\mathrm{DBA} / 2$ equivalent by the luciferase reporter assay showed significantly greater reporter activity of B6 than DBA222 (Fig. 2E). BLAST analysis of the additional DBA/2 sequence identified it as B2 SINE, the highly common family of repeats $[18,23,24]$ that are retrotransposons from RNA polymerase III-driven genes [25]. This data demonstrated that the presence of the transposable element in the DBA/2 core promoter inhibited transcription and suggested that the B2 SINE in the 5'-UTR of DBA/2 might contribute to polymorphisms in ALAS1 expression.

\section{Systematic analysis of DBA/2 B2 SINE sequence}

Studies by Espinoza et al. [26] of mouse B2 SINE RNA induced after heat shock have shown that it can act in trans by binding to Pol II and disrupting assembly of pre-initiation complexes at promoter regions. Prediction of the secondary structure of this B2 SINE 178mer non-coding RNA showed two duplex regions with 5' and 3' single stranded loops. Comparison of two deletion mutants suggested that both the consensus duplex regions were 
required for inhibitory activity whereas the single stranded tail regions were not. However, the $210 \mathrm{nt}$ found in the DBA/2 promoter contains additional nucleotides at the 3 ' end (CCCAGAG-) with low homology to B2 SINE and therefore the predicted secondary structure (RNAfold web server, http://rna.tbi.univie.ac.at/) of the whole insert differs from that suggested earlier, although similar in the presence of a 3' half loop ( Fig. $3 \mathrm{~A}$ and B). To test if the additional small sequence modulates the inhibitory effect of the entire transposon and to examine the importance of specific regions of the ALAS1 B2 SINE repeat, we constructed a panel of 10 deletion mutants (ranging from 22-70 nt deleted) across the 210 nt sequence in the DBA construct (Fig. 3C), which encompassed both single stranded loops and duplex stem regions. Comparison of the promoter activity of these constructs in situ in a cellular system showed that the whole of the 2B SINE is necessary to inhibit transcription (Fig. 3D), in contrast to studies using extracellular 2B SINE RNA and Pol II [26,27]. Why does only the full length DBA/2 B2 SINE element have an effect?

An ability of a transposon to modulate gene expression via the mechanism of binding transcription factors was suggested for the B1 SINE in a study which compared a canonical repeat with a B1 containing proximal binding sites for the transcription factor Slug and the carcinogen-activated dioxin receptor xenobiotic responsive element (XRE) [28]. We analyzed the additional sequence of the transposon present in DBA strain by MatInspector (http://www.genomatix.de/ ) and identified 71 putative binding sites for transcription factors and other regulatory proteins, including those which are known to repress gene expression. However, the transposon analysed here does not act in this manner since deletion of any fragment (from sizes of $\sim 20 \mathrm{nt}$ to $70 \mathrm{nt}$ ) throughout the repeat abolished its repression effect on the reporter gene expression.

As the complete sequence of the $210 \mathrm{nt}$ is required to inhibit the expression of reporter our findings support the mechanism of inhibition whereby B2 SINE RNA binds to Pol II and 
represses transcript synthesis [26]. Although the regions of B2 SINE identified as critical for Pol II binding were reported the mechanism of inhibition has not been completely clarified $[26,27,29]$ and the repeat was analysed out of context of the actual genomic sequence. In our experiments the transposon was the part of the $\sim 1000 \mathrm{nt}$ promoter construct and was analysed in an intracellular system with the flanking sequences present. The fact that the entire sequence is required for transcription repression emphasises the complexity of the regulatory event by 2B SINE non-coding RNA. The stability of the complex is also probably important for an inhibitory effect on transcription.

\section{Conclusions}

In summary, we have identified a distinct difference between inbred C57BL/6J and DBA/2 mice in hepatic mRNA levels of the gene for ALAS1, the main rate- controlling enzyme in nonerythroid heme synthesis. A polymorphism in the 5'-UTR consisting of a B2 SINE retrotransposon in the DBA/2 ALAS1 gene caused a marked reduction of promoter activity in a reporter assay compared to C57BL/6. Systematic deletions across the $210 \mathrm{nt}$ insert had no effect suggesting that the entire sequence was required for an inhibitory effect. These findings show that in mice the presence of the B2 SINE can introduce polymorphism in transcription of a gene. In the case of the ALASI this contributes to strain differences in the complex regulation of expression with a possible consequence for resistance or susceptibility to porphyria.

\section{Acknowledgements}

We thank the Protein and Nucleic Acid Laboratory in the University of Leicester and the Dr T. Gant laboratory for help.

\section{References}

[1] K. Furuyama, K. Kaneko and P. D. Vargas, Heme as a magnificent molecule with multiple missions: heme determines its own fate and governs cellular homeostasis, Tohoku J. Exp. Med. 213 (2007) 1-16. 
[2] M. A. Gilles-Gonzalez and G. Gonzalez, Heme-based sensors: defining characteristics, recent developments, and regulatory hypotheses, J. Inorg. Biochem. 99 (2005) 1-22.

[3] S. Hou, M. F. Reynolds, F. T. Horrigan, S. H. Heinemann and T. Hoshi, Reversible binding of heme to proteins in cellular signal transduction, Acc. Chem. Res. 39 (2006) 918-924.

[4] T. Chernova, J. R. Steinert, C. J. Guerin, P. Nicotera, I. D. Forsythe and A. G. Smith, Neurite degeneration induced by heme deficiency mediated via inhibition of NMDA receptor-dependent extracellular signal-regulated kinase 1/2 activation, J. Neurosci. 27 (2007) 8475-8485.

[5] M. Faller, M. Matsunaga, S. Yin, J. A. Loo and F. Guo, Heme is involved in microRNA processing, Nat. Struct. Mol. Biol. 14 (2007) 23-29.

[6] R. G. Hu, H. Wang, Z. Xia and A. Varshavsky, The N-end rule pathway is a sensor of heme, Proc. Natl. Acad. Sci. U. S. A. 105 (2008) 76-81.

[7] A. G. Roberts and G. H. Elder, Alternative splicing and tissue-specific transcription of human and rodent ubiquitous 5-aminolevulinate synthase (ALAS1) genes, Biochim. Biophys. Acta 1518 (2001) 95-105.

[8] J. Zheng, Q. Tian, W. Hou, J. A. Watts, L. W. Schrum and H. L. Bonkovsky, Tissuespecific expression of ALA synthase-1 and heme oxygenase- 1 and their expression in livers of rats chronically exposed to ethanol, FEBS Lett. 582 (2008) 1829-1834.

[9] S. Kakizaki, Y. Yamamoto, A. Ueda, R. Moore, T. Sueyoshi and M. Negishi, Phenobarbital induction of drug/steroid-metabolizing enzymes and nuclear receptor CAR, Biochim. Biophys. Acta 1619 (2003) 239-242.

[10] D. J. Fraser, A. Zumsteg and U. A. Meyer, Nuclear receptors constitutive androstane receptor and pregnane $\mathrm{X}$ receptor activate a drug-responsive enhancer of the murine 5-aminolevulinic acid synthase gene, J. Biol. Chem. 278 (2003) 39392-39401.

[11] A. S. Guberman, M. E. Scassa, L. E. Giono, C. L. Varone and E. T. Canepa, Inhibitory effect of AP-1 complex on 5-aminolevulinate synthase gene expression through sequestration of cAMP-response element protein (CRE)-binding protein (CBP) coactivator, J. Biol. Chem. 278 (2003) 2317-2326.

[12] C. Handschin, J. Lin, J. Rhee, A. K. Peyer, S. Chin, P. H. Wu, U. A. Meyer and B. M. Spiegelman, Nutritional regulation of hepatic heme biosynthesis and porphyria through PGC-1alpha, Cell 122 (2005) 505-515.

[13] K. Kaasik and C. C. Lee, Reciprocal regulation of haem biosynthesis and the circadian clock in mammals, Nature 430 (2004) 467-471.

[14] L. Claudio, T. Lee, M. S. Wolff and J. G. Wetmur, A murine model of genetic susceptibility to lead bioaccumulation, Fundam. Appl. Toxicol. 35 (1997) 84-90. 
[15] S. W. Robinson, B. Clothier, R. A. Akhtar, A. L. Yang, I. Latour, C. Van Ijperen, M. F. Festing and A. G. Smith, Non-Ahr gene susceptibility loci for porphyria and liver injury induced by the interaction of 'dioxin' with iron overload in mice, Mol. Pharmacol. 61 (2002) 674-681.

[16] S. R. Gross and J. J. Hutton, Induction of hepatic delta-aminolevulinic acid synthetase activity in strains of inbred mice, J. Biol. Chem. 246 (1971) 606-614.

[17] J. A. Goodrich and J. F. Kugel, Non-coding-RNA regulators of RNA polymerase II transcription, Nat. Rev. Mol. Cell. Biol. 7 (2006) 612-616.

[18] J. P. Kalkkila, F. R. Sharp, I. Karkkainen, M. Reilly, A. Lu, K. Solway, M. Murrel and J. Honkaniemi, Cloning and expression of short interspersed elements B1 and B2 in ischemic brain, Eur. J. Neurosci. 19 (2004) 1199-1206.

[19] T. A. Allen, S. Von Kaenel, J. A. Goodrich and J. F. Kugel, The SINE-encoded mouse B2 RNA represses mRNA transcription in response to heat shock, Nat. Struct. Mol. Biol. 11 (2004) 816-821.

[20] H. Nishihara, A. F. Smit and N. Okada, Functional noncoding sequences derived from SINEs in the mammalian genome, Genome Res. 16 (2006) 864-874.

[21] E. R. Garcia-Trevijano, M. L. Martinez-Chantar, M. U. Latasa, J. M. Mato and M. A. Avila, NO sensitizes rat hepatocytes to proliferation by modifying Sadenosylmethionine levels, Gastroenterology 122 (2002) 1355-1363.

[22] R. Davies, B. Clothier, S. W. Robinson, R. E. Edwards, P. Greaves, J. Luo, T. W. Gant, T. Chernova and A. G. Smith, Essential role of the AH receptor in the dysfunction of heme metabolism induced by 2,3,7,8-tetrachlorodibenzo-p-dioxin, Chem. Res. Toxicol. 21 (2008) 330-340.

[23] K. L. Bennett, R. E. Hill, D. F. Pietras, M. Woodworth-Gutai, C. Kane-Haas, J. M. Houston, J. K. Heath and N. D. Hastie, Most highly repeated dispersed DNA families in the mouse genome, Mol. Cell. Biol. 4 (1984) 1561-1571.

[24] D. A. Kramerov, S. V. Tillib, A. P. Ryskov and G. P. Georgiev, Nucleotide sequence of small polyadenylated B2 RNA, Nucl. Acids Res. 13 (1985) 6423-6437.

[25] M. Dewannieux and T. Heidmann, L1-mediated retrotransposition of murine B1 and B2 SINEs recapitulated in cultured cells, J. Mol. Biol. 349 (2005) 241-247.

[26] C. A. Espinoza, T. A. Allen, A. R. Hieb, J. F. Kugel and J. A. Goodrich, B2 RNA binds directly to RNA polymerase II to repress transcript synthesis, Nat. Struct. Mol. Biol. 11 (2004) 822-829.

[27] C. A. Espinoza, J. A. Goodrich and J. F. Kugel, Characterization of the structure, function, and mechanism of B2 RNA, an ncRNA repressor of RNA polymerase II transcription, RNA 13 (2007) 583-596. 
[28] A. C. Roman, D. A. Benitez, J. M. Carvajal-Gonzalez and P. M. Fernandez-Salguero, Genome-wide B1 retrotransposon binds the transcription factors dioxin receptor and Slug and regulates gene expression in vivo, Proc. Natl. Acad. Sci. U. S. A. 105 (2008) 1632-1637.

[29] H. Kettenberger, A. Eisenfuhr, F. Brueckner, M. Theis, M. Famulok and P. Cramer, Structure of an RNA polymerase II-RNA inhibitor complex elucidates transcription regulation by noncoding RNAs, Nat. Struct. Mol. Biol. 13 (2006) 44-48. 
Table 1. Constructs and primers used for sequencing.

$\begin{array}{ll}\text { Constructs } & \text { Primers } \\ \text { DBA222 and BL6 clones } & \text { 222F2 } \times 222 \mathrm{R} 1 \\ \Delta 70 \mathrm{~A} & 70 \mathrm{AF} 2 \times 70 \mathrm{AR} 2 \\ \Delta 70 \mathrm{~B} & 70 \mathrm{BF} 2 \times 70 \mathrm{BR} 2 \\ \Delta 70 \mathrm{C} & 70 \mathrm{CF} 2 \times 70 \mathrm{CR} 2 \\ \Delta \mathrm{A} & 26 \mathrm{AF} \times 70 \mathrm{AR} 2 \\ \Delta \mathrm{C} & 26 \mathrm{CF} \times 26 \mathrm{CR} \\ \Delta \mathrm{D} & 26 \mathrm{DF} \times 26 \mathrm{DR} \\ \Delta \mathrm{E} & 70 \mathrm{BF} 2 \times 27 \mathrm{ER} \\ \Delta \mathrm{FG} & 70 \mathrm{CR} 2 \times 31 \mathrm{GF} \\ \Delta \mathrm{G} & 31 \mathrm{GF} \times 31 \mathrm{GR} \\ \Delta \mathrm{H} & 70 \mathrm{CF} 2 \times 21 \mathrm{HR}\end{array}$

Primer sequences

$\begin{array}{ll}\text { MPRmiF } & \text { CGCGCTCGAGCGATTGCAGGCGGGC } \\ \text { MPRR2 } & \text { CGCGGAAGCTCTGCGCGGGCTCTCGA } \\ \text { MPRF2 } & \text { CGCGCTCGAGGCTTGCTTCAGTTGCTCGC } \\ \text { MPREx2R } & \text { CGCGAAGCT CGGCGAACGACAGTCTCC } \\ 222 F 2 & \text { ACGCGTACGTACCAAAGTTGGAGGCCA } \\ \text { 222R1 } & \text { GCTAGCTGCGCGGGCTCTCGACGAAG } \\ \text { 70AF2 } & \text { CCGGGAATTCCAGCAACCACATGGTG } \\ \text { 70AR2 } & \text { CCGGGAATTCGCTCTGGGTTTATTTTGAGAC } \\ \text { 70BF2 } & \text { CCGGGAATTCGAAGACAGCTACAGTGTACTTACA } \\ \text { 70BR2 } & \text { GGCCGAATTCCTGGGATTTGAACTCCG } \\ \text { 70CF2 } & \text { CCGGGAATTCAATAACTTATAGCTGTTC } \\ \text { 70CR2 } & \text { CGCGGAATTCGTCTTCAGACACTCCAGAAG } \\ 26 A F & \text { GCGCGAATTCTAAGAGCACCCGACTGC } \\ 26 \mathrm{CF} & \text { CCGGGAATTCACATGGTGGCTCACAACC } \\ \text { 26CR } & \text { CGCGGAATTCTTTGGAAGAGCAGTCGGG } \\ 26 \mathrm{DF} & \text { CGCGGAATTCGAGATCTGACTCCCTCTTCTGG } \\ 26 \mathrm{DR} & \text { CGCGGAATTCGGTTGCTGGGATTTGAAC } \\ 27 \mathrm{ER} & \text { CCGGGAATTCGTTACAGATGGTTGTGAGCCAC } \\ 31 \mathrm{GF} & \text { GGCCGAATTCCATTAAAAAAAAAACCCAGAGC } \\ \text { 31GR } & \text { CCGGGAATTCGTAAGTACACTGTAGCTGTCTTCAGAC } \\ 21 \mathrm{HR} & \text { CCGGGAATTCGTTTTATTATTATTATTATTTATTTATTATATG } \\ \end{array}$


Table 2. Relative hepatic expression of genes for enzymes of the heme synthesis pathway in C57BL/6J and DBA/2 mice as estimated by gene array analysis.

\begin{tabular}{lcc}
\hline Enzyme & Gene symbol & $\begin{array}{c}\text { Ratio of DBA/2 } \\
\text { relative to C57BL/6J }\end{array}$ \\
\hline 5-Aminolevulinic acid synthase 1 & $A L A S 1$ & $0.46^{*}$ \\
5-Aminolevulinic acid dehydratase & $A L A D$ & $3.33^{*}$ \\
Hydroxymethylbilane synthase & $H M B S$ & 1.12 \\
Uroporphyrinogen III synthase & $U R O S$ & 1.01 \\
Uroporphyrinogen decarboxylase & $U R O D$ & 0.96 \\
Coproporphyrinogen oxidase & $C P O$ & 1.00 \\
Protoporphyrinogen oxidase & $P P O X$ & 0.67 \\
Ferrochelatase & $F E C H$ & 1.05 \\
\hline
\end{tabular}

Results are means of hepatic cDNA from 4 or $5 \mathrm{C} 57 \mathrm{BL} / 6 \mathrm{~J}$ mice hybridized with random choice cDNAs from DBA/2 mice. For some genes more than one EST clone was present on the arrays and those shown represent the median example. $*$ C57BL/6J results were significantly different from DBA/2 $(\mathrm{p}<0.05)$. 
Figure legends

Fig. 1. Levels of ALAS1 mRNA in whole liver (A) and isolated hepatocytes (B) of C57BL/6J and DBA/2 mice. Relative mRNA levels were determined by qRT-PCR with $\beta$-actin as the reference gene. Results are the mean $+\mathrm{SD}$ for 3-5 samples. ALAS1 expression in DBA/2 levels was significantly lower than in C57BL/6J $(\mathrm{p}<0.05)$

Fig. 2. Comparison of constructs of the 5' region of ALAS1 gene from C57BL/6 mice. (A) Regions encompassing constructs. (B) Comparison of relative activity of the promoter constructs in a luciferase reporter assay \pm SD for 3-5 assays per construct. (C) Activity of the 2MPR construct in control cells and those treated with TCPOBOP, phenobarbital and DMSO detected by reporter assay. (D) Comparison of the size of the 2MPR construct from C57BL/6J with that amplified from DBA/2 mice. (E) Activity of the promoter constructs of the C57BL/6 region -989 to $+81 \mathrm{nt}$ compared with the DBA/2 equivalent in the luciferase reporter assay.

Fig. 3. Effects of systematic deletions in the additional $210 \mathrm{nt}$ on luciferase reporter activity. (A) Predicted secondary structures of the B2 SINE 203 nt and of (B) the whole sequence (210 nt) in DBA strain (http://rna.tbi.univie.ac.at/). (C) Comparison of B6 and DBA222 constructs and schematic representation of systematic deletions in DBA222 construct. (D) Promoter activity of the B2 SINE deletion constructs in the luciferase reporter assay relative to B6 and DBA222. Results are means of 3-5 assays \pm SD.

* Significantly different from B6 $(\mathrm{p}<0.05)$. 
A

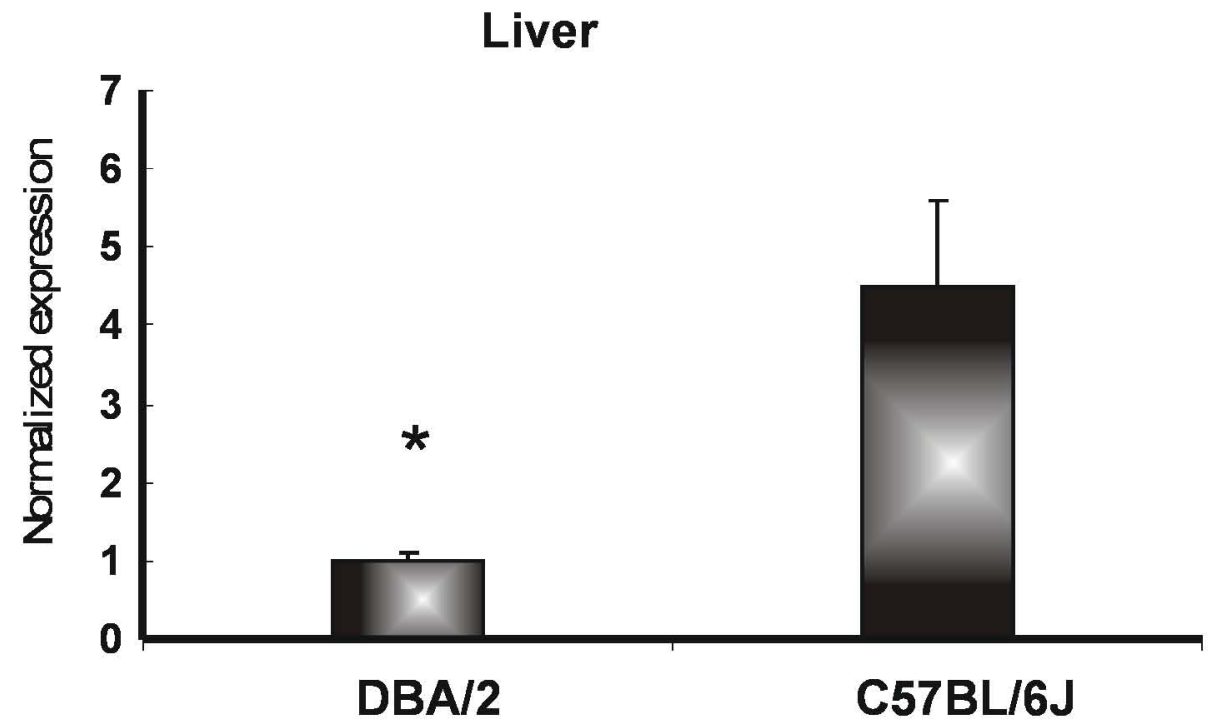

B

Hepatocytes

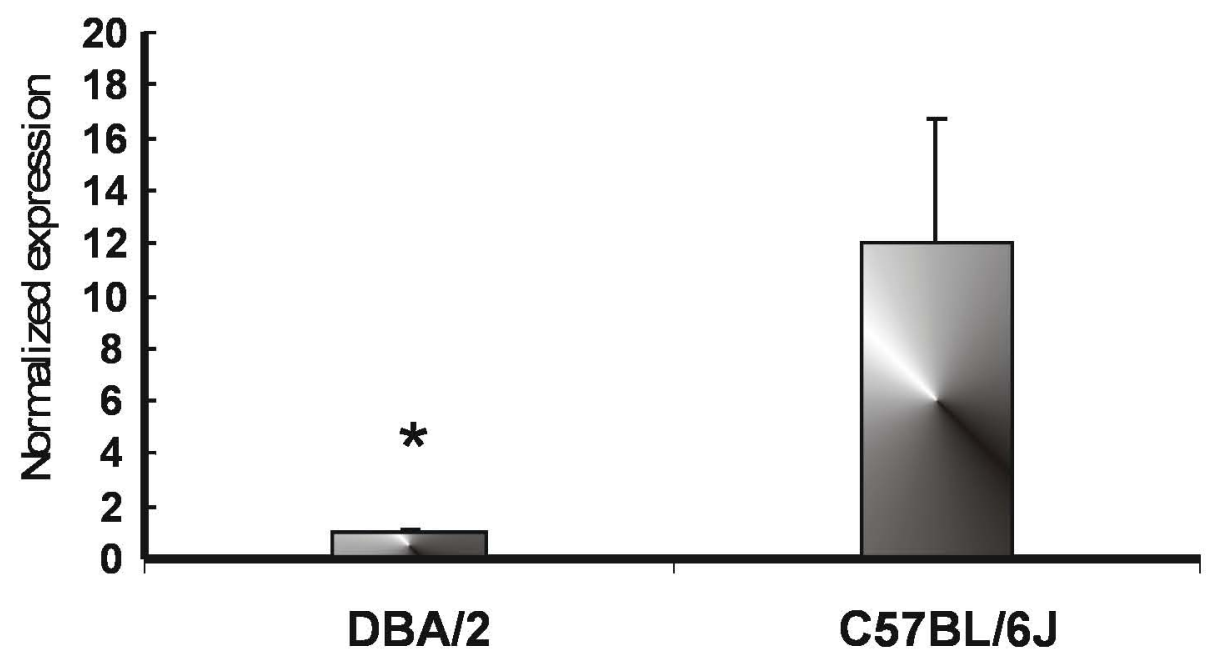


A

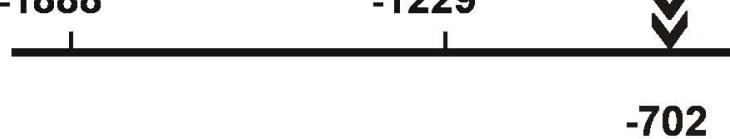

TATA

ATG

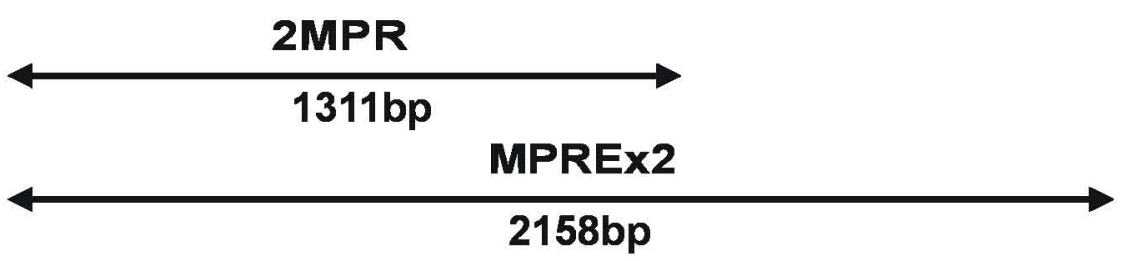

MPRM

1968bp

B

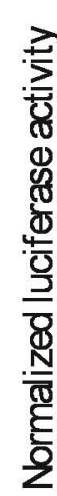

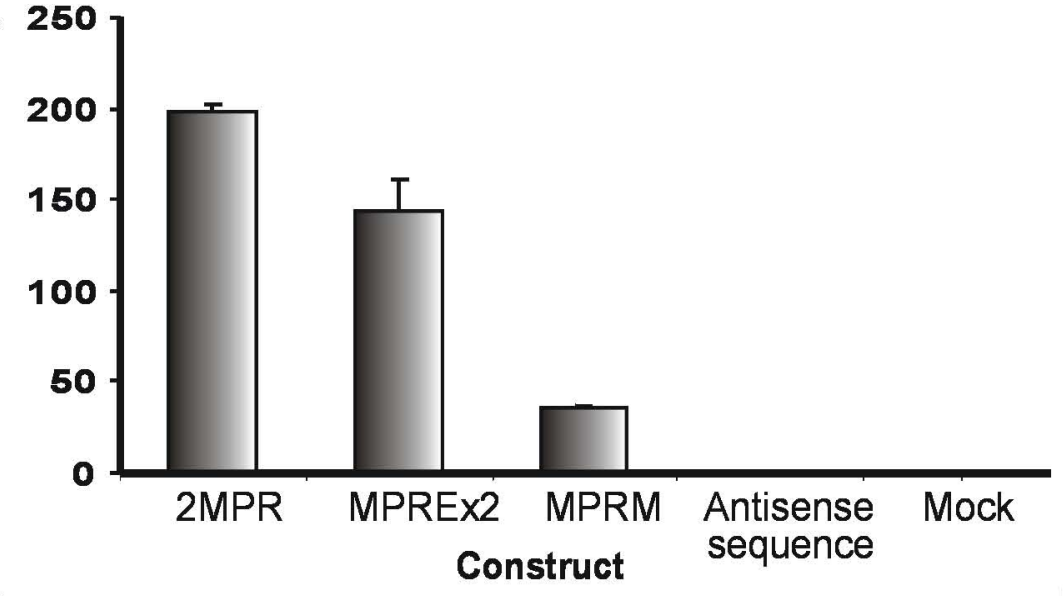

DBA/2 BI6

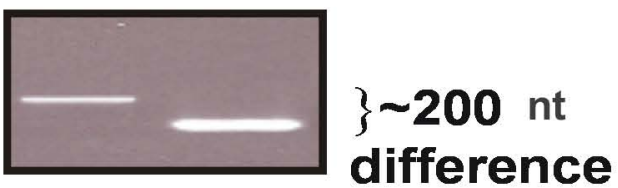

C

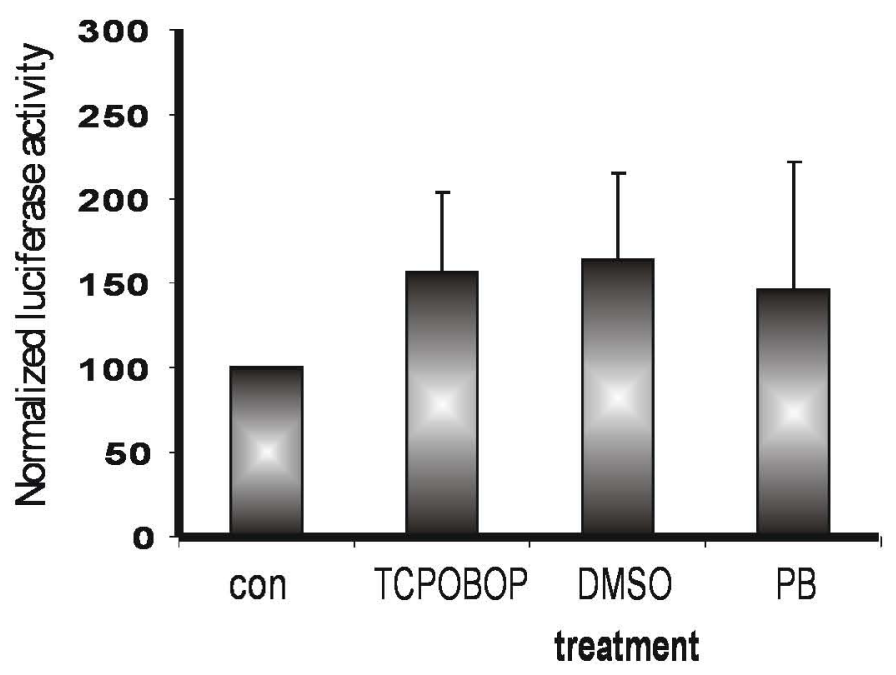

E

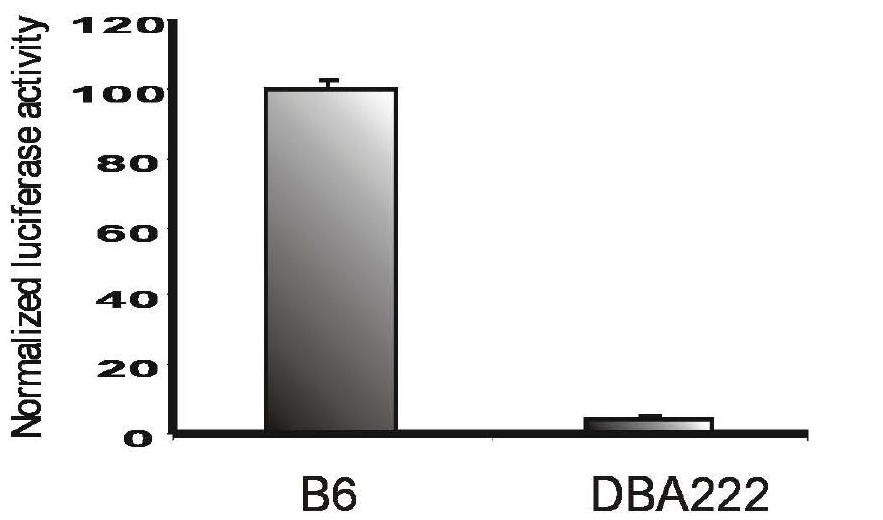




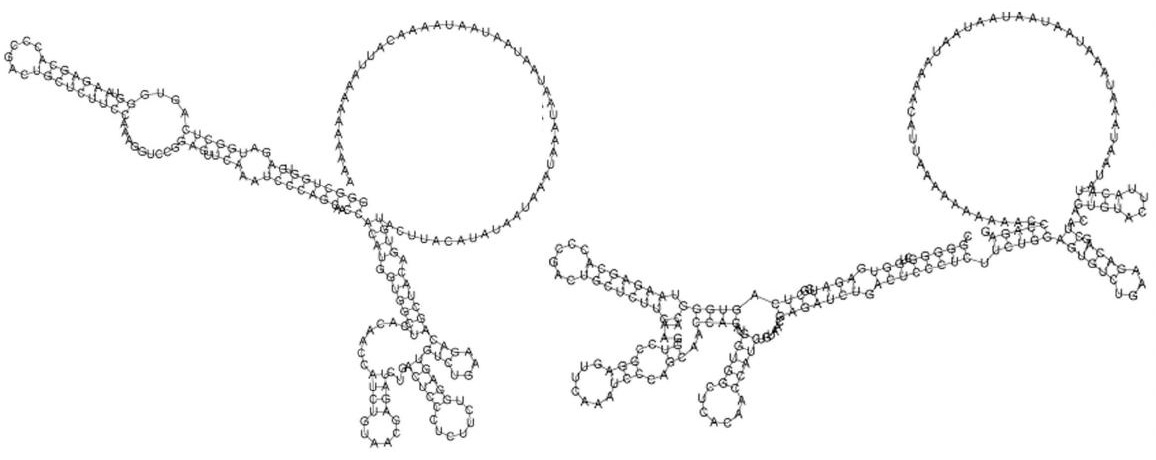

C

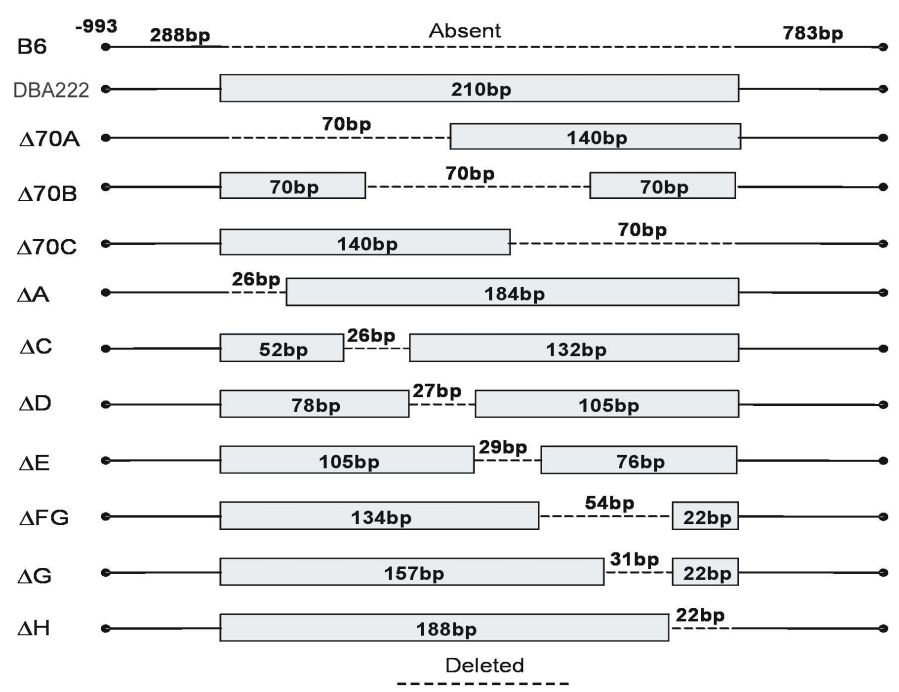

D

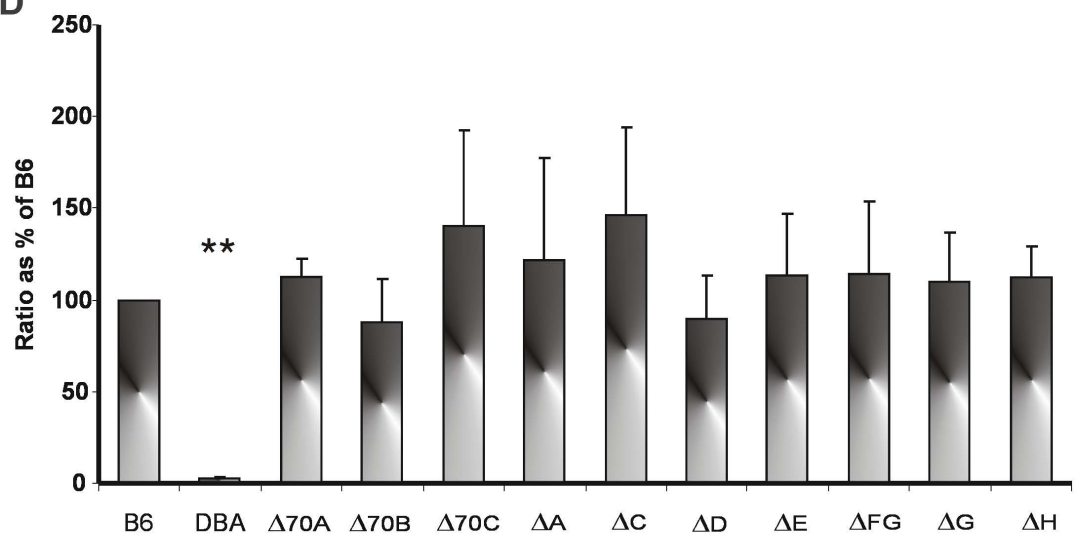

Construct 